\title{
Ocorrência de Ascaris suum em suínos abatidos na Zona da Mata, Minas Gerais
}

\author{
Occurence of the Ascaris suum in slaughtered swine in Zona da Mata region, Minas \\ Gerais State
}

\author{
Anderson Silva DIAS ${ }^{1,2}$; Alexander Malbar TANURE'; Hugo Granato Vieira Cardoso MANHÃES ${ }^{1}$ \\ ${ }^{1}$ Curso de Medicina Veterinária da Faculdade de Castelo, Castelo-ES, Brasil \\ ${ }^{2}$ Departamento de Medicina Veterinária da Universidade Federal de Viçosa, Viçosa-MG, Brasil
}

\begin{abstract}
Resumo
Os suínos são acometidos por diversas enfermidades e as verminoses se constituem como as principais causas de não ganho de peso, competição e diminuição da conversão alimentar, descarte de vísceras ao abate e perdas financeiras. O nematoide Ascaris suum se constitui na principal parasitose na suinocultura nacional. Esse trabalho teve como objetivo avaliar o nível de infestação por A. suum em suínos em idade de abate provenientes de granjas tecnificadas localizadas na região do município de Piranga, região oeste da Zona da Mata, Minas Gerais, latitude 2045'45”S, longitude $43^{\circ} 18^{\prime} 10^{\prime \prime} \mathrm{W}$. Os animais foram abatidos em três matadouros localizados no município de Piranga, e foram observados os tratos gastrintestinais dos suínos abatidos quanto à presença desse parasito. Foi observada a presença do nematoide em animais provenientes de $90 \%$ das granjas e a ocorrência total foi $22,4 \%$, média de todo o período experimental. Também foi observado que, durante o período experimental, a ocorrência de A. suum no lume intestinal dos animais não apresentou diminuição e os protocolos de everminação não foram eficazes para a diminuição da ocorrência desses helmintos nas propriedades onde foram empregados.
\end{abstract}

Palavras-chave: Ascaris suum. Ocorrência. Suínos. Abate.

\begin{abstract}
Swine are affected by several diseases and worms constitute the main causes of absence of weight gain, competition and decreased feed conversion, discards of slaughter viscera and financial losses. The nematode Ascaris suum remains a major parasitic disease in national livestock pigs. The objective of this work was to evaluate the level of infestation by $A$. suum in pigs at slaughter age provenient from intensive production systems located in Zona da Mata region, latitude $20^{\circ} 45^{\prime} 45^{\prime \prime} \mathrm{S}$, longitude $43^{\circ} 18^{\prime} 10^{\prime \prime} \mathrm{W}$, in tropical Southeastern, Brazil. The animals were slaughtered at three slaughterhouses located in the municipality of Piranga, and their gastrointestinal tracts were observed in order to identify the presence of those helminthes. We observed the presence of nematode in animals from $90 \%$ of the farms and the total occurrence was $22.4 \%$, media of all the experimental period. It was also observed that, during the experimental period, the occurrence of A. suum in the gastrointestinal tube did not present reduction and the protocol to administer anthelmintic were not efficient to decrease the occurrence this helminthes in proprieties in which they were applied.
\end{abstract}

Keywords: Ascaris suum. Occurrence. Swine. Slaughter.

\section{Introdução}

Dentre os parasitos mais frequentes na suinocultura, Ascaris suum constitui um dos principais ${ }^{1,2}$. A presença e a persistência desse helminto no meio devem-se, principalmente, a sua capacidade de sobrevivência na forma de ovo larvado, estrutura bastante resistente às condições ambientais e altamente capaz de reinfectar os animais ${ }^{3}$. A densidade populacional, o estresse térmico, a ineficiência na limpeza nas instalações onde os animais vivem e as condições climáticas (como temperaturas altas e umidade do ambiente) são fatores que predispõe mais facilmente a contaminação dos animais ${ }^{4}$.

De acordo com Polley e Mostert ${ }^{5}$, Freitas ${ }^{6}$ e Urquhart et al. ${ }^{4}$, A. suum possui distribuição mundial e parasita os suínos principalmente nos primeiros meses de vida. De acordo com Roepstorff e Nansen ${ }^{7}$ e Ramos,

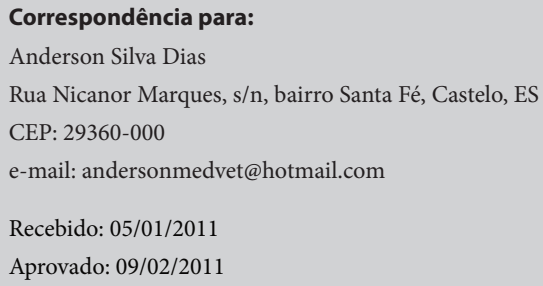


Faustino e Oliveira Filho ${ }^{8}$, a existência de resistência anti-helmíntica e a capacidade de propagação do parasito, devido à prolificidade das fêmeas, contribuem para aumentar a persistência desse agente dentre os animais. Deve-se considerar ainda que, de acordo com Lignon et al. ${ }^{3}$, as formulações anti-helmínticas deveriam ser testadas quanto à eficácia com certa periodicidade para evitar que ocorram problemas associados a sua eficácia. Os mesmos autores afirmam que esse procedimento deveria ser realizado por órgãos de fiscalização governamentais. Dada a importância desse helminto, deve-se lembrar que ele, ainda que raramente, é capaz de causar larva migrans visceral no homem, apresentando, portanto, caráter zoonótico ${ }^{4,9}$.

As perdas econômicas associadas ao A. suum estão relacionadas à condenação do fígado dos suínos abatidos, que uma vez infectado apresenta manchas branco leitosas, caracterizando alterações de aspecto repugnante ${ }^{1}$, além de o agente ser considerado carreador de Actinobacillus e vírus influenza para o fígado e de coronavírus respiratório para os pulmões, com possibilidades de disseminação desses agentes para o rebanho ${ }^{10}$. Esse helminto também é considerado responsável por atraso no ganho de peso dos animais, o que atrasa a destinação desses para o abate, desencadeando aumento dos custos de produção com alimentação e medicação ${ }^{7}$.

O agente é transmitido para os leitões principalmente no nascimento e quando da amamentação na creche; as infecções podem persistir mesmo após tratamento devido à ineficácia do tratamento e reinfecções ${ }^{5}$.

Diante do exposto, o presente estudo teve como objetivo avaliar a ocorrência de A. suum em suínos provenientes de granjas e abatidos em matadouros em Piranga, MG.

\section{Material e Método}

Durante o período de março de 2005 e outubro de 2006, foram examinados os intestinos de 2719 suínos abatidos em três matadouros, localizados na zona rural do Município de Piranga (latitude 20 $45^{\prime} 45^{\prime \prime}$, longitude $43^{\circ} 18^{\prime} 10^{\prime \prime} \mathrm{W}$ ), oeste da região da Zona da Mata, Minas Gerais. Esses animais, mestiços de cruzamento industrial, foram fornecidos por dez granjas da região que possuem sistema de produção intensiva tecnificada. Dentre os suínos, 96\% deles (2610 animais) eram jovens, com idade ao redor de cinco meses, 1128 desses eram fêmeas e 1482, machos castrados e 109 animais (em torno de 4\%) eram fêmeas adultas.

Esses animais, criados nas granjas e destinados ao abate, eram vermifugados entre os 50 e 70 dias e as matrizes, com periodicidade de dois meses, com febendazole (4\%, durante dez dias). Uma vez diagnosticada a presença de $A$. suum no intestino desses animais durante o abate, as granjas das quais esses eram oriundos realizavam um protocolo de everminação que consistia em administrar o fármaco em todos os lotes de animais na propriedade com repetição após 20 dias. O vermífugo utilizado na ração foi o febendazole (4\%), em 250 g por tonelada, durante dez dias.

Os suínos enviados para os matadouros eram registrados no livro de entradas, que era constituído de dados dos lotes de animais como; o nome da granja, a idade, o peso e o sexo. No dia do abate, cada suíno era identificado por número, lote e granja de origem. Nos três matadouros, os procedimentos eram uniformes e consistiam em, durante o abate e evisceração; os intestinos eram enviados para a triparia, onde todo o conteúdo presente dentro do órgão era expulso por compressão digital cuidadosa ao longo de toda a extensão dos intestinos, de forma a ocorrer a eliminação completa do que estava presente no lume intestinal e, assim, os helmintos, quando presentes, eram expelidos, contados e enviados para identificação de acordo com a chave classificatória de Yamaguti ${ }^{11}$, no laboratório de Parasitologia, do Departamento 
de Medicina Veterinária da Universidade Federal de Viçosa, Minas Gerais.

Os dados foram tabulados de acordo com a procedência dos animais, o mês de abate e entre animais positivos e negativos quanto à presença de adultos de A. suum no lume intestinal, que quando presentes eram quantificados. Foi realizada uma análise estatística descritiva das médias mensais para a ocorrência do agente. Para averiguar se houve diferença de ocorrência de A. suum entre suínos machos e fêmeas foi realizado o teste de qui quadrado a 0,05 de nível de significância.

\section{Resultados e Discussão}

Das dez granjas de suínos que forneceram animais para o abate durante o período experimental, nove (90\%) apresentaram resultados positivos para presença de Ascaris suum, o único helminto encontrado no lume intestinal dos suínos. Dos 2719 animais avaliados durante esse período experimental, 599 (22,40\%) apresentaram a presença desse helminto. Não foi possível afirmar que houve diferença na ocorrência desse helminto em animais de diferentes granjas porque o número de animais fornecidos por cada granja era diferente e sofriam flutuações a cada mês (Tabela 1).

Algumas granjas, como a número 02 e 07 (Figura 1), apresentaram o percentual de animais positivos de $36,84 \%$ e $43,41 \%$, respectivamente, ocorrência alta, comparada às outras granjas $(01,03,04,05,06$, 08, 09 e 10). Os protocolos de everminação de reforço aplicado nas granjas (que consistiam em repetir a administração do vermífugo após 20 dias, em iguais períodos de everminação por dez dias seguidos em todos os lotes de animais da propriedade) quando era detectada a presença de $A$. suum nos animais ao abate possibilitam uma discussão a respeito da eficácia dos fármacos (febendazole, $4 \%, 250 \mathrm{mg} /$ toneladas). Sabese que apenas uma fêmea desse helminto é capaz de lançar 200 mil ovos por dia e as baias, mesmo com a desinfecção, dificilmente seriam completamente

Tabela 1 - Relação de suínos abatidos divididos por meses, por granja e quanto à presença ou ausência de Ascaris suum no lume intestinal. Na última linha, encontra-se o total de animais positivos e negativos quanto à presença de A. suum no lume intestinal ao abate, divididos por granja, durante todo o período experimental. Os animais foram abatidos em três matadouros localizados na zona rural do município de Piranga, Zona da Mata, Minas Gerais, durante o período de março de 2005 a outubro de 2006

\begin{tabular}{|c|c|c|c|c|c|c|c|c|c|c|c|c|c|c|c|c|c|c|c|c|c|}
\hline \multicolumn{22}{|c|}{ Animais abatidos por mês } \\
\hline \multirow{3}{*}{ Mês } & \multicolumn{21}{|c|}{ Granjas } \\
\hline & \multicolumn{2}{|c|}{01} & \multicolumn{2}{|c|}{02} & \multicolumn{2}{|c|}{03} & \multicolumn{2}{|c|}{04} & \multicolumn{2}{|c|}{05} & \multicolumn{2}{|c|}{06} & \multicolumn{2}{|c|}{07} & \multicolumn{2}{|c|}{08} & \multicolumn{2}{|c|}{09} & \multicolumn{2}{|c|}{10} & \multirow[t]{2}{*}{ total } \\
\hline & + & - & + & - & + & - & + & - & + & - & + & - & + & - & + & - & + & - & + & - & \\
\hline $\operatorname{mar} / 05$ & 0 & 3 & 9 & 15 & 2 & 16 & 1 & 12 & 0 & 6 & 0 & 13 & 5 & 20 & 0 & 5 & 0 & 2 & 1 & 3 & 113 \\
\hline abr/05 & 0 & 1 & 13 & 10 & 1 & 8 & 0 & 4 & 0 & 7 & 1 & 7 & 11 & 9 & 1 & 2 & 0 & 7 & 0 & 5 & 87 \\
\hline mai/05 & 0 & 2 & 12 & 17 & 1 & 16 & 0 & 20 & 0 & 7 & 0 & 11 & 11 & 6 & 0 & 3 & 0 & 1 & 1 & 3 & 111 \\
\hline jun/05 & 0 & 7 & 9 & 19 & 3 & 39 & 0 & 19 & 0 & 8 & 0 & 8 & 29 & 24 & 0 & 3 & 0 & 3 & 0 & 1 & 172 \\
\hline $\mathrm{jul} / 05$ & 0 & 8 & 11 & 14 & 1 & 54 & 0 & 30 & 1 & 6 & 2 & 6 & 30 & 26 & 0 & 2 & 0 & 4 & 0 & 2 & 197 \\
\hline ago/05 & 0 & 7 & 5 & 10 & 2 & 28 & 0 & 16 & 0 & 7 & 0 & 7 & 41 & 40 & 1 & 3 & 0 & 5 & 1 & 2 & 175 \\
\hline set/05 & 0 & 8 & 11 & 23 & 2 & 7 & 2 & 17 & 0 & 8 & 0 & 13 & 13 & 15 & 0 & 2 & 0 & 7 & 0 & 3 & 131 \\
\hline out/05 & 0 & 6 & 4 & 18 & 1 & 31 & 0 & 15 & 0 & 5 & 0 & 5 & 16 & 23 & 0 & 4 & 0 & 4 & 0 & 2 & 134 \\
\hline nov/05 & 1 & 5 & 4 & 10 & 3 & 34 & 1 & 9 & 1 & 5 & 0 & 9 & 9 & 27 & 1 & 6 & 0 & 7 & 1 & 5 & 138 \\
\hline dez/05 & 0 & 1 & 13 & 18 & 0 & 51 & 0 & 15 & 0 & 8 & 1 & 8 & 26 & 59 & 0 & 2 & 0 & 3 & 0 & 2 & 207 \\
\hline $\mathrm{jan} / 06$ & 1 & 3 & 7 & 15 & 5 & 33 & 0 & 13 & 0 & 7 & 0 & 7 & 17 & 39 & 0 & 2 & 0 & 8 & 1 & 4 & 162 \\
\hline fev/06 & 0 & 1 & 12 & 17 & 0 & 31 & 1 & 12 & 0 & 8 & 0 & 12 & 24 & 45 & 0 & 3 & 0 & 5 & 0 & 2 & 173 \\
\hline $\mathrm{mar} / 06$ & 0 & 4 & 9 & 9 & 3 & 11 & 0 & 3 & 0 & 2 & 0 & 4 & 6 & 9 & 0 & 2 & 0 & 3 & 1 & 5 & 71 \\
\hline abr/06 & 1 & 3 & 11 & 11 & 0 & 12 & 0 & 12 & 0 & 6 & 1 & 6 & 7 & 19 & 0 & 3 & 0 & 5 & 0 & 2 & 99 \\
\hline mai/06 & 0 & 1 & 15 & 18 & 1 & 8 & 0 & 8 & 0 & 9 & 0 & 9 & 19 & 15 & 0 & 6 & 0 & 4 & 0 & 4 & 117 \\
\hline jun/06 & 1 & 1 & 9 & 26 & 1 & 29 & 1 & 24 & 0 & 7 & 0 & 15 & 15 & 15 & 1 & 2 & 0 & 2 & 0 & 3 & 152 \\
\hline jul/06 & 0 & 9 & 8 & 17 & 0 & 19 & 0 & 13 & 0 & 9 & 0 & 9 & 24 & 14 & 1 & 2 & 0 & 6 & 0 & 1 & 132 \\
\hline ago/06 & 0 & 3 & 11 & 22 & 0 & 10 & 0 & 16 & 0 & 9 & 1 & 9 & 12 & 14 & 0 & 3 & 0 & 6 & 0 & 3 & 119 \\
\hline set/06 & 0 & 9 & 12 & 15 & 3 & 7 & 0 & 13 & 1 & 7 & 0 & 7 & 14 & 10 & 0 & 1 & 0 & 2 & 1 & 3 & 105 \\
\hline out/06 & 0 & 10 & 4 & 20 & 2 & 8 & 1 & 18 & 0 & 5 & 1 & 7 & 17 & 22 & 0 & 2 & 0 & 3 & 0 & 4 & 124 \\
\hline total & 4 & 92 & 189 & 324 & 31 & 452 & 7 & 289 & 3 & 136 & 7 & 172 & 346 & 451 & 5 & 58 & 0 & 87 & 7 & 59 & 2719 \\
\hline
\end{tabular}


$\% 100$

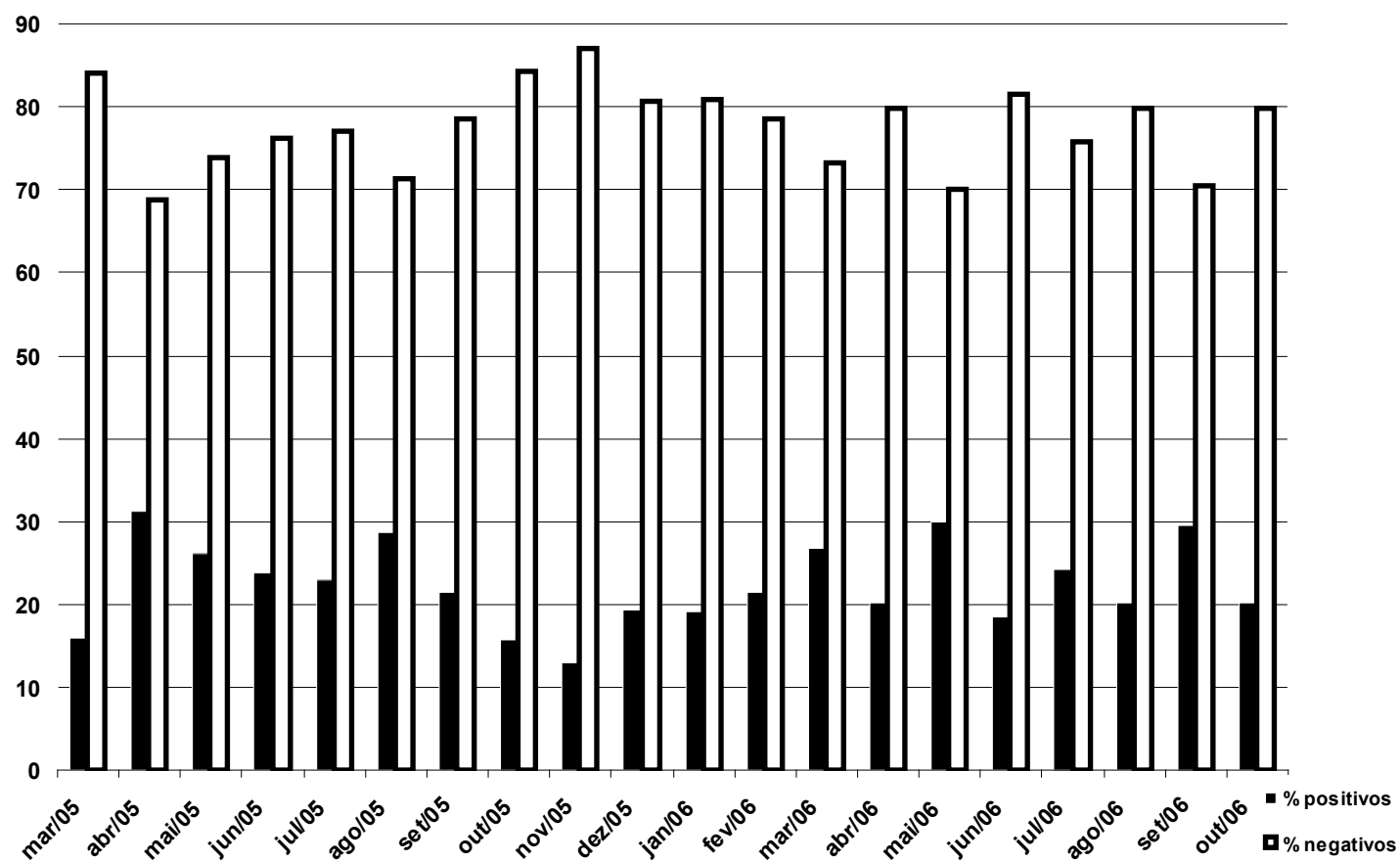

Figura 1 - Percentual de suínos abatidos, divididos mensalmente, quanto à presença ou ausência de Ascaris suum no lume intestinal. Os animais eram 2610 animais; 1128 eram fêmeas e 1482, machos castrados e 109 eram adultos (matrizes), e foram abatidos em três matadouros localizados na zona rural do município de Piranga, Zona da Mata, Minas Gerais, durante o período de março de 2005 a outubro de 2006

descontaminadas de ovos desse helminto, permitindo a reinfecção dos animais ${ }^{1,4,6}$. As amplas possibilidades de reinfecção dos animais tornam os protocolos de everminação passíveis de questionamento. Desse modo, esforços deveriam ser direcionados também para diminuir as formas infectantes do helminto presentes no meio, pois assim, a probabilidade de reinfecção seria também reduzida, o que possibilitaria a diminuição da frequência do uso de anti-helmíntico.

Não houve diferença $(\mathrm{P}>0,05)$ de infestação quanto ao sexo dos animais analisados (os animais machos eram castrados e foram criados nas mesmas condições que as fêmeas). Não foi observada a presença de A. suum no lume intestinal das matrizes destinadas ao abate (109 animais), o número de matrizes analisadas era baixo, além disso, elas eram vermifugadas bimestralmente nas granjas, e por serem animais adultos apresentam resistência à reinfecções ${ }^{3,6,7}$, porém, de acordo com Ramos, Faustino e Oliveira Filho ${ }^{8}$ e Roepstorff e Nansen ${ }^{7}$, suínos adultos podem apresentar a presença de adultos $A$. suum no lume intestinal. No presente trabalho, a ocorrência desse parasito foi observada apenas nos suínos com idade em torno de cinco meses (2610 animais).

Na comparação por mês da ocorrência de A. suum nos suínos não foi observada diferença ( $\mathrm{p}>0,01)$, o que indica ausência de variação sazonal, ou seja, as condições climáticas e das instalações são favoráveis para reinfecção dos animais durante todo o ano para essa região em estudo. Nas instalações, a umidade no piso é alta e muitas vezes não há insolação adequada nesse ambiente, situações que permitem a sobrevivência dos ovos por maior período. Não se observar diferença de presença de $A$. suum nos animais abatidos, analisados mensalmente, significa que o programa de vermifugação instalado nas granjas que monitoraram 
a presença do nematoide através do abate não foi eficaz na redução no número de animais parasitados.

Em média, todos os animais eram enviados para o abate com idade em torno de cinco meses. Quanto ao peso dos animais também não se observou diferença ao abate $(\mathrm{P}>0,01)$ entre parasitados e não parasitados. Isso pode ser explicado pelo fato dos animais serem vermifugados preventivamente, o que reflete na baixa carga parasitária dos animais com presença de adultos de A. suum no lume intestinal, porém, não desencadeou em diferença de peso ao abate entre os animais parasitados e não parasitados. Outra possível explicação é que esses animais são alimentados de forma balanceada, além de ser considerado o potencial genético dos animais avaliados nesse estudo para ganho de peso eficiente. Em animais não advindos do cruzamento industrial e criados em sistema de produção extensiva, observam-se indivíduos com retardo no ganho de peso quando parasitados ${ }^{8,12,13}$, porém, as condições de criação dos animais analisados nesse estudo são de sistema tecnificado.

De acordo com Roepstorff e Nansen ${ }^{7}$, Humbert e Henry $^{13}$, Jesus e Miller ${ }^{12}$, D'Alencar et al. ${ }^{14}$ e Hoff, Silva e Monteiro ${ }^{15}$, a densidade populacional tem uma influência marcante sob a intensidade parasitária dos suínos, e espera-se que os sistemas de criação intensivos permitam maior nível de infestação dos animais. Nesse trabalho, observou se que a densidade populacional nas granjas está em torno de 1,6 animais por metro quadrado em média, um valor mediano, se comparado com sistemas de criações mais intensivos.

A alimentação com bagaço de cana para as marrãs (observada na granja número 02 ) pode ser considerada um fator favorável à reinfecção dos animais. Nessa propriedade, a cana era cultivada próxima aos galpões e a possibilidade desse solo e água estarem contaminados por ovos de A. suum não pode ser descartada.

Quanto à quantidade de nematoides encontrados no trato gastrintestinal, $51 \%$ dos animais parasitados apresentaram apenas um adulto no lume intestinal, sendo que em 39\% desses observou-se a presença de fêmeas. O percentual de animais parasitados que apresentavam um casal de nematoides foi de $23 \%$, observando-se que apenas em torno de um quarto dos animais infectados (5,6\% dos animais) apresentavam potencial, quando na idade de abate, para reinfectar o meio. $\mathrm{O}$ fato de se encontrar poucos nematoides no lume intestinal pode ser devido à resistência orgânica dos animais somada à ineficiência dos tratamentos anti-helmínticos e junto ao erro de manejo na desinfestação das instalações.

Um manejo integrado para o controle de verminoses em granja com taxas medianas de animais infectados ao abate deveria ser implementado e monitorado. Isso porque a presença desse parasito resulta em perdas econômicas significativas, acarretando um problema para a granja que pode se tornar incontrolável e ser responsável por gastos cada vez maiores com antihelmínticos e condenações em maior escala ao abate.

A detecção da presença de helmintos através do abate dos animais apresenta uma sensibilidade e especificidade alta, permitindo uma avaliação mais precisa, e pode ser útil, por exemplo, como nesse estudo, para direcionar os protocolos de everminação onde os animais são criados. Assim, comparando os dados apresentados nesse trabalho (ocorrência de 22,4\%) com aqueles obtidos por exames coproparasitológico, como o realizado por D’Alencar et al. ${ }^{14}$, na qual foi observado um percentual de 0,09\% de suínos parasitados por A. suum, pode-se dizer que, além de mais prático, permite o diagnóstico de animais parasitados por poucos helmintos ou de adultos jovens ou adultos que não estão liberando ovos quando as fezes são coletadas. Entretanto, não se pode esquecer que o exame coproparasitológico permite a detecção de helmintos nos animais em períodos anteriores à idade de abate, o que permite o tratamento prévio e evita maiores perdas.

Por meio desse estudo foi possível avaliar a real eficácia dos tratamentos empregados nos animais. Esse 
estudo apresentou-se como uma oportunidade para os suinocultores e profissionais assistencialistas do ramo obter dados e avaliar a eficácia dos protocolos de everminação empregados em seus animais.

\section{Conclusão}

Pode-se dizer que suínos abatidos na região do presente estudo apresentaram índice de infestação parasitária por Ascaris suum que não apresentou diminuição durante o período em que foi analisado (março de 2005 a outubro de 2006), e que esses protocolos de everminação de reforço empregados nas granjas não foram

\section{Referências}

1.BERNARDO, T. M.; DOHOO, I. R.; OGILVIE, T. Critical assessment of abattoir surveillance as a screening test for swine ascariasis. Canadian Journal Veterinary Research, v. 54, n. 2, p. 274-277, 1990.

2.SOUZA, A. C. M.; SOUSA, D. P.; NEVES, M. L. M. W. Frequência de infecções por helmintos em suínos criatórios urbanos na região de Recife-PE. In: JORNADA DE ENSINO, PESQUISA E EXTENSAO, 2004, Recife. Anais... Recife: UFRPE, 2004. CD-ROM.

3. LIGNON, G. B.; SOBESTIANSKY, J.; MORES, N.; GUIDONI; A. L. Ação do oxibendazole frente à migração de larvas de Ascaris suum em suínos. Comunicado Técnico, EMBRAPA, CNPSA, 1985. Disponível em: <http:/ /www.cnpsa.embrapa. br/ct/86/embrapa-cnpsa,abril>. Acesso em: 09 out. 2008.

4. URQUHART, G. M.; ARMOUR, J.; DUNCAN, J. L.; DUNN, A. M.; JENNINGS, F. W. Parasitologia veterinária. 2. ed. Rio de Janeiro: Guanabara Koogan, 1998. 273 p.

5. POLLEY, L. R.; MOSTERT, P. E. Ascaris suum in saskatchewan pigs: an abattoir survey of prevalence and intensity of infection. Canadian Veterinary Journal, v. 21, n. 11, p. 307-309, 1980.

6. FREITAS, M. G. Helmintologia veterinária. 6. ed. Belo Horizonte: Precisa, 1982. 253 p.

7. ROEPSTORFF, A.; NANSEN, P. Epidemiology and control of helminth infection in pigs under intensive and non-intensive production systems. Veterinary Parasitology, v. 54, n. 1/3, p. 69-85, 1994.

8. RAMOS, C. A. N.; FAUSTINO, M. A. G.; OLIVEIRA FILHO, E. F. Levantamento de parasitos gastrintestinais de suínos criados na região Metropolitana de Recife e Zona da Mata do Estado do Pernambuco. In: CONGRESSO DE INICIAÇÃO CIENTÍFICA, 12., 2002, Recife. Anais... Recife: UFRPE, 2002. p. 202-203. eficazes para diminuir a infecção dos animais por esse helminto, possivelmente devido a erros de manejo. Programas de controle integrado poderiam ser implementados e monitorados nas granjas para prevenir a reinfecção dos suínos e o uso da cana na alimentação das matrizes deveria ser contraindicado nessa ocasião.

\section{Agradecimentos}

Os autores agradecem a Secretaria Municipal de Agricultura e Meio Ambiente do Município de Piranga, MG, a Faculdade de Castelo, Castelo, Espírito Santo, a Fapemig e a Capes e ao CNPq.

9. ROLDÁN, W. H.; ESPINOZA, Y. A.; HUAPAYA, P. E.; HUIZA, A. F.; SEVILLA, C. R.; JIMÉNEZ, S. Frequency of human toxocariasis in a rural population from Cajamarca, Peru determined by dot-ELISA test. Revista do Instituto de Medicina Tropical de São Paulo, v. 51, n. 2, p. 67-71, 2009.

10.CLAEREBOUT, E.; VERCRUYSSE, J.; ERRICO, P.; VLAMINCK, J. Developing of the vaccine against Ascaris suum. Merelbeke, Belgium. Laboratory of Parasitology Faculty of Veterinary Medicine: Ghent University. 2009. Disponível em: <http://www.vetparasitology.ugen t.be/page1/ page24/ page24.html.> Acesso em: 28 ago. 2009.

11.YAMAGUTI, S. Systhema helminthium: the nematodes of vertebrates. New York: The Interscience Publishers, 1961. $679 \mathrm{p}$.

12.JESUS, L. P.; MILLER, G. Helmintos do estômago de suínos na região de Pelotas. Revista Brasileira de Agrociểncia, v. 6, n. 2, p. 181-187, 2000.

13.HUMBERT, J. F.; HENRY, C. Studies on the prevalence and transmission of lung and stomach nematodes of the wild boar (Sus scrofa) in France. Journal Wildlife Disease, v. 25, n. 3, p. 335-341, 1989.

14.D'AlENCAR, A. S.; FAUSTINO, M. A. G.; SOUSA, D. P.; LIMA, M. M.; ALVES, L. C. Infecção por helmintos e coccídios em criação de suínos de sistema confinado localizada no município de Macaragibe-PE. Ciência Veterinária Tropical, v. 9, n. 2/3, p. 79-86, 2006.

15.HOFF, G.; SILVA, A. S.; MONTEIRO, S. G. Avaliação do parasitismo e comparação de técnicas de análise fecal em suínos de granjas da região oeste do estado de Santa Catarina. Revista da Faculdade de Zootecnia, Veterinária e Agronomia, v. 12, p. 106-115, 2005. 